\title{
INCLUSIVE JET PRODUCTION AT THE TEVATRON
}

\author{
OLGA NORNIELLA* \\ Institut de Física d'Altes Energies, \\ Edifici Cn. Facultat Ciències UAB, \\ E-08193 Bellaterra (Barcelona), SPAIN
}

Preliminary results on inclusive jet production in proton-antiproton collisions at $\sqrt{s}=1.96 \mathrm{TeV}$ based on $1 \mathrm{fb}^{-1}$ of CDF Run II data are presented. Measurements are preformed using different jet algorithms in a wide range of jet transverse momentum and jet rapidity. The measured cross sections are compared to next-toleading order perturbative QCD calculations.

The measurement of the inclusive jet cross section is an important test of perturbative QCD (pQCD) predictions over more than 8 orders of magnitude, probing distances down to $10^{-19} \mathrm{~m}$. The increased center-of-mass energy in Run II (from $1.8 \mathrm{TeV}$ to $1.96 \mathrm{TeV}$ ), the highly upgraded CDF detector $^{1}$, and the amount of data collected allow the performance of jet measurements in an extended region of jet transverse momentum, $p_{T}^{\text {jet }}$, and jet rapidity, $y^{j e t}$. Jet measurements at large rapidities are important because they constrain the parton densities in the proton in a kinematic region where signal for compositeness is not expected.

This contribution presents preliminary results on inclusive jet production based on $1 \mathrm{fb}^{-1}$ of CDF Run II data with jets defined using the midpoint algorithm ${ }^{2}$ and the longitudinally invariant $k_{T}$ algorithm ${ }^{3}$. These algorithms, unlike the Run I cone-based algorithm ${ }^{4}$, are infrared and collinear safe to all orders in $\mathrm{pQCD}$, making possible a better comparison between data and theory. The midpoint algorithm is a cone-based algorithm that uses midpoints between a pair of jets as additional seeds to make the clustering infrared safe. The algorithm includes a prescription to solve the situation with overlapping cones and merges them whenever the common $p_{T}$ is bigger than a fixed fraction of the $p_{T}$ of the less energetic jet. To emulate this experimental merging/splitting feature, the pQCD calculations

*On behalf of the CDF Collaboration. 
increase the cone size through an ad hoc parameter $R_{\text {sep }}$. The $k_{T}$ algorithm, inspired by QCD parton radiation, successively clusters particles according to their relative transverse momentum and contains a parameter, $\mathrm{D}$, that approximately controls the size of the resulting jets. The $k_{T}$ algorithm has the advantage that it does not need to solve situations with overlapping jets. A previous measurement using the $k_{T}$ algorithm at the Tevatron during Run $\mathrm{I}^{5}$ observed a marginal agreement with $\mathrm{NLO}$ pQCD at low $p_{T}^{\text {jet }}$, thus suggesting the $k_{T}$ algorithm was particularly challenging in hadron collisions. However, a recently published result by the CDF experiment, based on data collected during Run II period ${ }^{6}$, shows that this apparent discrepancy is removed after non-perturbative corrections are included.

Figure 1 shows the measured inclusive jet cross sections using the $k_{T}$ algorithm with $\mathrm{D}=0.7$, for jets with $p_{T}^{\text {jet }}>54 \mathrm{GeV} / \mathrm{c}$ in five jet rapidity regions up to $\left|y^{\text {jet }}\right|=2.1$. For presentation, the different cross sections are scaled by a given factor. The measured cross sections have been corrected for detector effects back to the hadron level using PYTHIA-Tune A Monte Carlo ${ }^{7}$, that provides an accurate description of the underlying event ${ }^{8}$ and jet shapes ${ }^{9}$ in Run II. The cross sections decrease over more than seven orders of magnitude as $p_{T}^{\text {jet }}$ increases. The systematic uncertainties on the data, mainly dominated by a $2 \%$ to $3 \%$ uncertainty in the jet energy scale, vary from $10 \%$ at low $p_{T}^{\text {jet }}$ to about $50 \%$ at high $p_{T}^{\text {jet }}$. The measurements are compared to $\mathrm{pQCD}$ NLO predictions as determined using JETRAD ${ }^{10}$ with CTEQ6.1M PDFs ${ }^{11}$ and renormalization and factorization scales set to $p_{T}^{\max } / 2$. The theoretical calculations include correction factors, $C_{H A D}$, to take into account non-perturbative effects related to the underlying event and fragmentation processes. The factors have been evaluated with PYTHIA-Tune A, as the ratios between the nominal cross sections at the hadron level and the ones obtained after turning off multiple parton interactions between remnants and fragmentation into hadrons. The difference obtained when HERWIG ${ }^{12}$ is used instead of PYTHIA has been taken as systematic uncertainty in the correction factors. The ratios between the measurements and the theory show a good agreement over all $p_{T}^{\text {jet }}$ ranges in all rapidity regions. The uncertainty in the theoretical prediction is dominated by the uncertainty on the gluon PDF at high $\mathrm{x}$ which, at high $p_{T}^{\text {jet }}$, goes from ${ }_{-30}^{+70} \%$ to ${ }_{-40}^{+140} \%$ for central and forward jets, respectively. The already smaller uncertanties in the data compared to that in the NLO pQCD calculations show that the measurements will contribute to a better knowledge of the parton distributions inside the proton. 

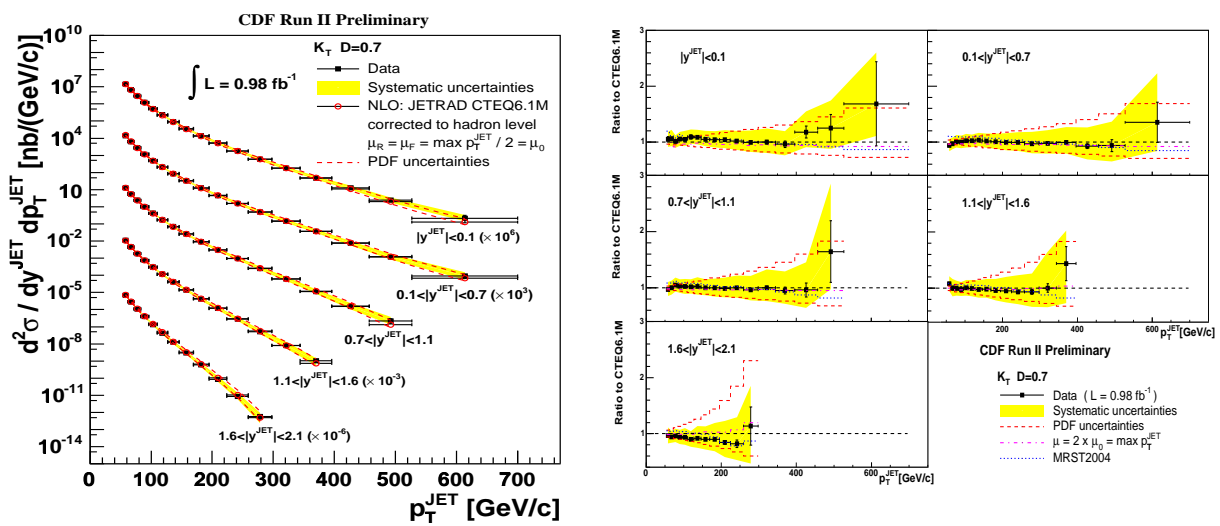

Figure 1. Left: Inclusive jet cross sections measured using the $k_{T}$ algorithm with $\mathrm{D}=0.7$ for jets with $p_{T}^{\text {jet }} \geq 54 \mathrm{GeV} / \mathrm{c}$ in five rapidity regions up to $\left|y^{j e t}\right|=2.1$. The black squares represent the measured cross sections and the shaded bands indicate the total systematic uncertainty on the data. The measurements are compared to pQCD NLO calculations. The dashed lines represent the PDF uncertainties on the theoretical predictions. Right: Ratios between the measured and theoretical inclusive jet cross sections.

For central jets, $0.1<\left|y^{j e t}\right|<0.7$, the measurements are repeated using a $\mathrm{D}$ parameter equal to 0.5 and 1.0. As $\mathrm{D}$ increases, the average size of the jet in $\eta-\phi$ space increases, and the measurement becomes more sensitive to underlying event contributions. The good agreement still observed between the measured cross sections and the NLO pQCD predictions indicates that the soft contributions are well under control.

Figure 2 presents the results using the midpoint algorithm for central jets. The measurement is in a good agreement with NLO pQCD calculations, which are obtained with EKS program using $R_{s e p}=1.3$ with CTEQ6.1M PDFs and renormalization and factorization scales set to $p_{T}^{j e t} / 2$.

In summary, this contribution reports preliminary results on inclusive jet production in proton-antiproton collisions at $\sqrt{s}=1.96 \mathrm{TeV}$, based on $1 \mathrm{fb}^{-1}$ of CDF Run II data, using different jet search algorithms. The measurements are in a good agreement with NLO pQCD calculations. In particular, for central jets and at high $p_{T}^{\text {jet }}$ no deviation with respect to the theory is found. In the most forward region, the total systematic uncertainty on the data is smaller than that on the theoretical calculations. Therefore, these new results will contribute to a better understanding of the gluon PDF at high $\mathrm{x}$ in the proton. 

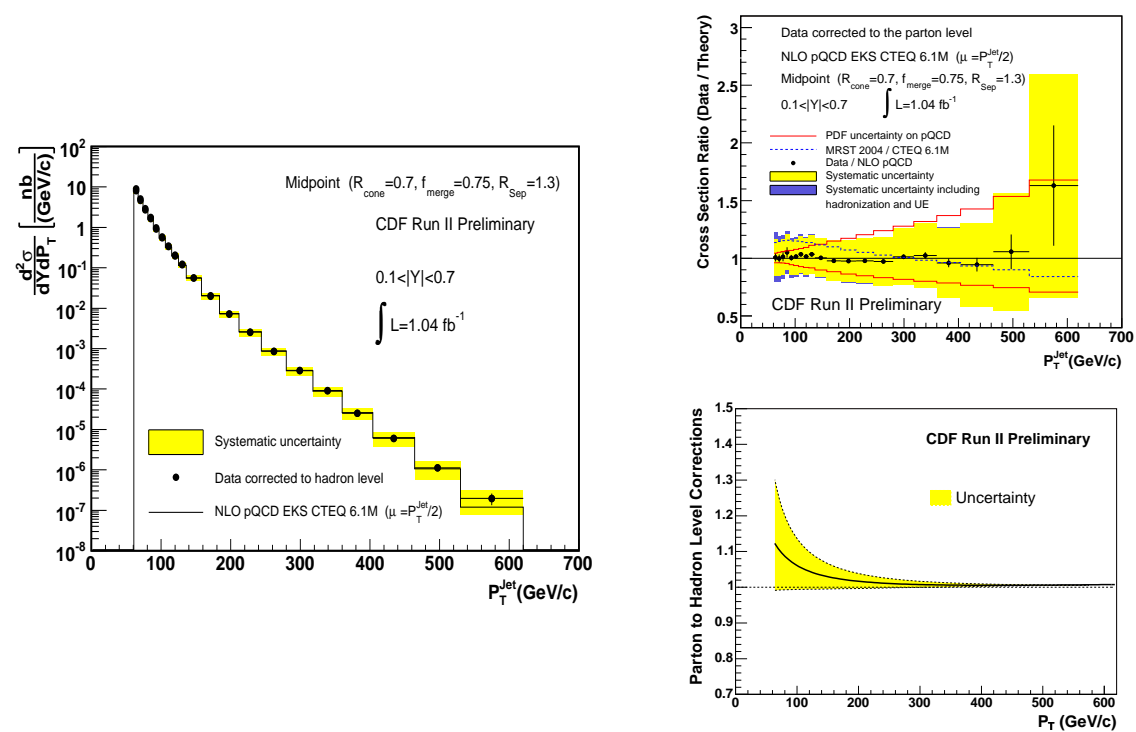

Figure 2. Left: Inclusive jet cross section measured using the midpoint algorithm for jets in the region $0.1<\left|y^{\text {jet }}\right|<0.7$. Top Right: Ratio of the measured and theoretical inclusive jet cross section. Bottom Right: Parton-to-hadron correction factors applied to the pQCD NLO calculations. The shaded band indicates the Monte Carlo modeling uncertainty.

\section{References}

1. R. Blair et al., CDF Collaboration, FERMILAB-Pub-96/390-E, (1996).

2. G. C. Blazey et al., hep-ex/0005012.

3. S. D. Ellis and D.E.Soper, Phys Rev., D48, 3160 (1993).

4. F. Abe et al, CDF Collaboration, Phys Rev., D45, 1148 (1992).

5. V. M. Abazov et al., D0 Collaboration, Phys. Lett., B 525, 211 (2002)

6. A. Abulencia et al., CDF Collaboration, Phys Rev. Lett., 96, 122001 (2006).

7. T. Sjostrand et al, Comput. Phys. Commun., 135, 238 (2001).

8. R. D. Field, $M E / M C$ Tuning Workshop., Fermilab, October 2002.

9. D. Acosta et al, CDF Collaboration, Phys Rev., D71, 112002 (2005).

10. W. T. Giele et al., Phys Rev. Lett., 73, 2019 (1994).

11. J. Pumplin et al., JHEP, 0207 (2002).

12. G. Corcella et al, JHEP, 0101, 010 (2001). 